\title{
The effect of the local field and dipole-dipole interactions on the absorption spectra of noble metals and the plasmon resonance of their nanoparticles
}

\author{
I.I. Shaganov \\ Vavilov State Optical Institute, 199034, St.-Petersburg, Russia \\ T.S. Perova \\ Trinity College Dublin \\ Kevin Berwick \\ Technological University Dublin, kevin.berwick@tudublin.ie
}

Follow this and additional works at: https://arrow.tudublin.ie/engscheceart

Part of the Electrical and Electronics Commons

\section{Recommended Citation}

Shaganov, II., Perova, T.S. \& Berwick, K. (2017). The effect of the local field and dipole-dipole interactions on the absorption spectra of noble metals and the plasmon resonance of their nanoparticles. Photonics and Nanostructures - Fundamentals and Applications, vol. 27, pp. 24-31. doi.org/10.1016/ j.photonics.2017.09.003.

This Article is brought to you for free and open access by the School of Electrical and Electronic Engineering at ARROW@TU Dublin. It has been accepted for inclusion in Articles by an authorized administrator of ARROW@TU Dublin. For more information, please contact arrow.admin@tudublin.ie, aisling.coyne@tudublin.ie, gerard.connolly@tudublin.ie.

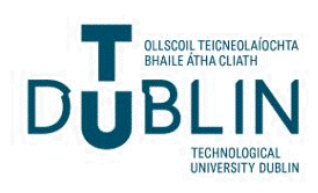




\title{
The effect of the local field and dipole-dipole interactions on the absorption spectra of noble metals and the plasmon resonance of their nanoparticles
}

\author{
I.I. SHAGANOV ${ }^{1}$, T.S. PEROVA ${ }^{2,3}$, AND K. BERWICK ${ }^{4}$ \\ ${ }^{I}$ Vavilov State Optical Institute, 199034, St.-Petersburg, Russia \\ ${ }^{2}$ Department of Electronic and Electrical Engineering, Trinity College Dublin, The University of Dublin, \\ Dublin 2, Ireland. E-mail: perovat@tcd.ie. Tel: +35318961432. \\ ${ }^{3}$ ITMO University, 49 Kronverkskiy pr., Saint Petersburg, 197101, Russia \\ ${ }^{4}$ School of Electrical and Electronic Engineering, Dublin Institute of Technology, Dublin 8, Ireland
}

\section{Research Highlights}

- The impact of dipole-dipole interactions on the absorption spectra of noble metals and their nanoparticles is investigated

- The calculations of the noble metal $(\mathrm{Ag}, \mathrm{Au}$ and $\mathrm{Cu})$ micro-characteristics are presented

- The difference in the frequency of the resonant absorption of electron oscillators localized in the nanoparticle and the intrinsic frequency of the free electrons in the bulk metal is due to resonant dipole-dipole interactions

\begin{abstract}
Based on the Drude-Lorentz model, the effect of free and bound electrons on the optical properties of noble metals and their nanoparticles is analyzed. It is shown that the shifts of absorption bands of plasmons localized in spherical nanoparticles with respect to the zero frequency of free electrons in a bulk metal can be estimated on the basis of the theory of resonant dipole-dipole interactions. The calculation includes account for differences between the effective and average electromagnetic fields. It is established that the difference in oscillator strength for free electrons in the bulk metal, obtained using the Drude-Lorentz model, and the microscopic oscillators in the corresponding spherical nanoparticle, is due to background polarization. This occurs at the expense of high-frequency excitation of the bound electrons. These results show that interparticle interactions in the noble metals in the quasi-static approximation can be regarded as dipole-dipole interactions of point dipoles with a concentration equal to the concentration of free electrons.
\end{abstract}

\section{INTRODUCTION}

The investigation of the optical properties of metal nanoparticles of various sizes and shapes has attracted a great deal of attention from researchers over the last two decades (see, for example, Refs. [1-5]). Excitation of surface plasmons in these particles has stimulated considerable interest in these materials, since their unique optical properties can be exploited in a variety of devices [6-8]. Possible applications include highly sensitive chemical and biological sensors [9-11], single particle/molecule detection [12], surface enhanced spectroscopy [13,14], waveguiding [4] and lasing [5]. This research has resulted in a number of textbooks and comprehensive reviews, some of which are cited above, devoted to the synthesis and fabrication of a variety of composite systems as well as modelling and characterisation of their linear and nonlinear optical properties. Modelling of the optical properties of small particles is under active development using a modification of the Maxwell-Garnett approximation [2,15] using numerical calculation techniques [16]. 
A somewhat different approach was undertaken recently, as described in Ref. [17], where the influence of dipole-dipole interactions on the spectral properties of noble metal nanoparticles was investigated. In addition, the dependence of noble metal nanoparticle spectra on the properties of the surrounding medium was explored. In this study, the possibility of using a combination of the theory of intermolecular interactions and dispersion of effective field (DEF) approaches for the analysis of the effect of resonant and inductive-resonant dipole-dipole interactions on the plasmon absorption frequency of materials is investigated. Both granular films and colloidal solutions of gold and silver nanoparticles were considered. It was shown that theoretical calculations, assuming a quasi-static approximation applies, are in good agreement with experimental data. Note that resonant dipole-dipole interactions in this context refer to the interaction of similar oscillators in a single-component or composite environment $[17,18]$. Inductive-resonant interactions reflect the influence of a transparent matrix on the spectral characteristics of an isolated particle and are apparent in the absorption spectra of dilute composite media, see [17] and references therein. Based on this approach, it is shown in present study that the difference between the frequency of the Lorentz oscillator, describing the absorption of free electrons in the bulk metal, and the absorption frequency of the plasmon, localized within the corresponding spherical nanoparticle, is due to interparticle interactions of free electrons. These interactions occur both between the electrons and between electrons and positive lattice ions. This latter interaction can be considered a dipole-dipole interaction of point oscillators, whose concentration is equal to the number of atoms per unit volume in a metal. It will be shown that the differences in the oscillator strength of the free-electron oscillators in bulk metal obtained using the Drude-Lorentz model and microscopic oscillators in the corresponding spherical nanoparticles are due to background polarization occurring at the expense of high-frequency excitation of bound electrons.

\section{THEORETICAL APPROACH}

\section{Models used for the description of the optical characteristics of metals}

The theoretical basis for the description of the optical characteristics of metals was laid down in the classic work by Drude (see, for example, Refs. [1,2,19,20]), who considered the conduction electrons as a free electron gas, excluding the effect of interband transitions involving the bound electrons. In order to allow for interband transitions, which have a significant impact on the optical properties of metals in the near IR, visible and ultraviolet regions of the spectrum, the integrated Drude-Lorentz (DL) $[1,2,19,20]$ model was introduced. In this model, the dielectric function of the metal includes two terms. These two terms take into account contributions from both conduction electrons and bound electrons involved in interband transitions.

In accordance with the DL model, the complex dielectric permittivity, $\varepsilon(\omega)$, of the metal can be expressed as a contribution of intraband effect (or free electron effect, Drude formula), $\varepsilon_{\text {intra }}(\omega)$, and interband effect (or bound electron effect, Lorentz formula), $\varepsilon_{\text {inter }}(\omega)$, in the following form $[1,2,19,20]$

$$
\varepsilon(\omega)=\left(\varepsilon_{\text {intra }}(\omega)\right)+\left(\varepsilon_{\text {inter }}(\omega)\right)=\left(1-\frac{F_{0} \omega_{p}^{2}}{\left[\omega\left(\omega-i \Gamma_{0}\right)\right]}\right)+\left(\sum_{j=1}^{k} \frac{F_{j} \omega_{p}^{2}}{\left[\left(\omega_{j}^{2}-\omega^{2}\right)+i \omega \Gamma_{j}\right]}\right) .
$$

Here, $\omega_{p}$ is the plasma frequency and the term $F_{0} \omega_{p}^{2}$ is related only to intraband transitions of the free electrons with oscillator strength $F_{o}$ and damping constant $\Gamma_{o}$. Similarly $F_{j}, \omega_{j}$ and $\Gamma_{j}$ represent the dispersion parameters of the $j$-th oscillator, describing the corresponding interband transition for the bound electrons. In general, Eqn. (1) does not contain a correction for the local field. This is taken into account only when considering the contribution of bound electrons to the dynamic matrix of transition metals [19]. In this case, it is assumed that a local (effective) field, $E_{\text {eff }}(\omega)$, acting on a free electron in a metal is equal to the mean $E_{a v}(\omega)$ field in this medium [1]. However, according to the results of Ref. [17], it appears that when applying the point-dipole approximation, allowing for the spectral differences between the $E_{\text {eff }}(\omega)$ and $E_{a v}(\omega)$ fields is wholly justified for metals. Indeed, free electrons in a metal are under the influence of both the external field and the fields created by other electrons and ions. In order to take into account electron-electron and electron-ion interactions, one must take into account the local field, which is the electrodynamic equivalent of these interactions in the metal. To a first approximation, this can be done on the basis of the Lorentz relation, which gives

$$
E_{\text {eff }}(\omega)=E_{a v}(\omega)[\varepsilon(\omega)+2] / 3,
$$

where $\varepsilon(\omega)=\varepsilon_{1}(\omega)-i \varepsilon_{2}(\omega)$ represents the spectrum of the complex dielectric constant of a condensed medium (which in the case considered here is a bulk metal as well as its nanoparticles). Since the dielectric function of the bulk metal, $\varepsilon^{b u l k}(\omega)$, which is determined experimentally, experiences the 
influence of several interparticle interactions, it is assumed that their effect on the spectroscopic characteristics of the metal can be taken into account using the above expression.

This assertion is supported by a series of studies by Granqvist et al. (see, for example Refs. $[18,21])$ as well as reports [22,23] published earlier, where a combination of an effective medium approach and dipole-dipole interactions considering the local-field effect was applied to the description of the optical properties of thin metal films and small metal particles. However, only average local field factors were used when considering the differences in the integrated intensities of the spectra of nanoparticles of different shapes by introducing the resultant depolarization factors.

In this study, it will be demonstrated how interparticle interactions manifest themselves in the optical properties of noble metals and their nanoparticles, based on a consideration of the spectral differences between $E_{e f f}(\omega)$ and $E_{a v}(\omega)$ and the theory of dipole-dipole interactions. As a consequence, it is clear that the free and bound electrons in these cases have different effects on the characteristics of the plasmon absorption bands.

Attempts have been made recently to more accurately predict the dielectric function of metals using various models, including a modified Debye model, a Drude-Lorentz model (DL) and of BrendelBormann (BB) model among others, see, for example, Refs. [24-26]. These efforts are necessary due to the escalating use of metal films and particles in various fields of science and technology [3-5,27-29] and the consequent requirement for more accurate predictions of their optical constants over a wide spectral range. For example, in Ref. [24], DL and BB models and experimental data was used to evaluate the dispersion parameters describing the dielectric function for a wide range of different metals, including $\mathrm{Au}, \mathrm{Ag}, \mathrm{Cu}$ and $\mathrm{Al}$, over a broad spectral range. In order to analyse the role of dipole-dipole interactions on the optical properties of bulk metals and the absorption bands of the corresponding localized plasmons, we used parameters from the dispersion oscillator in [24], describing the absorption contribution of free electrons to the dielectric function of $\mathrm{Au}, \mathrm{Ag}$ and $\mathrm{Cu}$.

The dispersion of the dielectric constant of condensed matter, $\varepsilon^{\text {bulk }}(v)$, in general can be represented as

$$
\varepsilon^{b u l k}(v)=\varepsilon_{\infty}+\sum_{j} \frac{\left[N e^{2} / \pi m c^{2}\right] \cdot F_{j}}{v_{j}^{2}-v^{2}+i \gamma_{j} v}
$$

where $N$ is the concentration of the oscillators in $\mathrm{cm}^{-3}, e$ and $m$ are the charge and mass of the electron, $c$ is the speed of light in vacuum and $v_{j}, F_{j}$ and $\gamma_{j}$ are the frequency, oscillator strength and damping constant of the $j$-th dispersion oscillator respectively. The sum on the right-hand side of this expression is similar to the last term in Eqn. (1). We will use the wavenumber $v$, as used in molecular spectroscopy, instead of $\omega$ below, but will continue to refer to it as frequency, measured in $\mathrm{cm}^{-1}$ and related to $\omega$ as $v=$ $\omega / 2 \pi c$.

Eqn. (2) for an isolated resonance in the visible or infrared region of the spectrum can be expressed in terms of microscopic quantities, taking into account the difference between $E_{\text {eff }}$ and $E_{a v}$ fields, leading to its modification in the form of [30,31]:

$$
\varepsilon^{b u l k}(v)=\varepsilon_{\infty}+\frac{\left[\left(N f_{j} e^{2}\right) / \pi m c^{2}\right] \cdot\left[\left(\varepsilon_{\infty}+2\right) / 3\right]^{2}}{v_{j}^{2}-\left[\left(N f_{j} e^{2}\right) / 3 \pi m c^{2}\right] \cdot\left(\varepsilon_{\infty}+2\right) / 3-v^{2}+i \gamma_{j} v} .
$$

Here $f_{j}, v_{j}$, and $\gamma_{j}$ are the oscillator strength, intrinsic (or eigen) frequency and damping constant of a particular $j^{\text {th }}$ microscopic oscillator, $N$ is their concentration, and $\varepsilon_{\infty}$ is the background dielectric constant in this spectral region, taking into account high-frequency transitions.

It is known that the Drude model can be considered a special case of a Lorentz model, in which the frequency of the oscillator, taking into account the contribution of the free electrons, is 0 (i.e., $v_{j}=v_{0}=$ 0 in Eqn.(2)) [19] and $\varepsilon_{\infty}=1$ (for the ideal electron gas), which results in term $\left(\varepsilon_{\text {intra }}(\omega)\right)$ in Eqn. (1), i.e. in Drude formula. The dielectric function of bulk metal in the low-energy region, where electronic intraband transitions dominate, can be described to a good approximation by Drude model with taking into account the contribution of intense interband transitions simply by the background permittivity $\varepsilon_{\infty}$ and, therefore, we can calculate $\varepsilon^{b u l k}(v)$ as

$$
\varepsilon^{\text {bulk }}(v)=\varepsilon_{\infty}+\frac{\left[\left(N e^{2}\right) / \pi m c^{2}\right] \cdot F_{0}}{v_{0}^{2}-v^{2}+i \gamma_{0} v}
$$


Here $v_{0}=0$ is the frequency of the macroscopic dispersion oscillator for free electrons with oscillator strength $F_{0}$, and damping constant $\gamma_{0}$. These parameters for $\mathrm{Ag}, \mathrm{Au}$ and $\mathrm{Cu}$ can be taken from the Ref. [24].

Our calculations show that the spectrum of imaginary part of dielectric function $\varepsilon_{2}^{\text {micro }}(v)$ of microscopic oscillator, correspondent to plasmonic resonance for noble metals, is dominant in the visible and infrared range. Therefore, to a certain extent, we can assume that the dielectric function for a bulk metal, $\varepsilon^{b u l k}(v)$, can be calculated using Eqns. (3) on the one hand, and on the other hand using Eqn. (3a), i.e.

$$
\varepsilon_{\infty}+\frac{\left[\left(N e^{2}\right) / \pi m c^{2}\right] \cdot F_{0}}{v_{0}^{2}-v_{0}+i \gamma_{0} v}=\varepsilon_{\infty}+\frac{\left[\left(N f_{c} e^{2}\right) / \pi m c^{2}\right] \cdot\left[\left(\varepsilon_{\infty}+2\right) / 3\right]^{2}}{v_{c}^{2}-\left[\left(N f_{c} e^{2}\right) / 3 \pi m c^{2}\right] \cdot\left(\varepsilon_{\infty}+2\right) / 3-v^{2}+i \gamma_{c} v},
$$

where $f_{c}, v_{c}$, and $\gamma_{c}$ are the oscillator strength, intrinsic frequency and damping constant of a particular microscopic electronic oscillator, and $N$ is their concentration, corresponding to the number of free electrons per unit volume. A comparison of both parts of Eqn. (3b) shows that the strength of the microscopic oscillator, $f_{c}$, is connected with the dispersion oscillator, $F_{0}$, by the expression

$$
f_{c}=F_{0} \theta_{a v}
$$

where

$$
\theta_{a v}=9 /\left(\varepsilon_{\infty}+2\right)^{2}
$$

is the mean value of the Lorentz polarization correction.

Analysis of Eqn. (3b) also shows that the frequency of the dispersion macroscopic oscillator $v_{0}$ and the intrinsic frequency of the microscopic oscillator, $v_{c}$, corresponding to the absorption frequency of the localized plasmon, are related by

$$
v_{c}^{2}-\left[\frac{N f_{c} e^{2}}{3 \pi m c^{2}}\right] \cdot \frac{\varepsilon_{\infty}+2}{3}=v_{0}^{2}=0 .
$$

Note that the above assumptions for comparing two parts in Eqn. (3b) were made only to find simple formulas Eqns. (4) and (5) for the relationship between the oscillators' strength and the frequencies of macroscopic and microscopic oscillators, which will be compared later in Tables 1 and 2 using other approaches as well.

It has been shown previously that the origin of the differences between the frequencies and intensities of optical macro- and micro-characteristics are the resonant interactions of molecules, or oscillating dipoles, in a condensed medium. Accounting for a dispersive effective local field (DEP) [32,33], as previously developed by Bakhshiev et al. [34,35], is a convenient technique for the analysis of the impact of these interactions on the absorption spectra of condensed media.

2. Accounting for the dispersive effective field in the spectroscopy of dipole-dipole interactions in a condensed medium

The DEP technique is a variant of the effective field method used in the derivation of Eqn. (3a), allowing the Lorenz model to take into account the difference in strength between the local and average fields in the condensed matter under consideration. This methodology is based on a comparison of the observed absorption spectrum of a condensed medium and the corresponding corrected spectrum, interpreted as a dielectric loss spectrum of its microscopic counterpart. In recent years, the efficacy of the DEP method has been demonstrated in the study of the spectral characteristics of granular metal films [36], as well as of condensed matter experiencing spatial restriction $[33,37]$.

Thus, the DEP method allows the establishment of a connection between the observed absorption spectra of condensed matter and the spectral characteristics of the microscopic oscillators responsible for this absorption. The effective, or acting, field $E_{\text {eff }}(v)$ plays the role of an electrodynamic equivalent of the total impact on the molecule, or particle, of the external macroscopic field and the force field of intermolecular interactions (IMI). The external macroscopic field $E(v)$ is considered as a weak perturbation and so this consideration does not go beyond standard linear molecular optics.

It should be noted that these considerations can be attributed not only to the individual molecules but also to the corresponding nanoparticles and clusters. Their absorption will be determined by the spectroscopic characteristics of their constituent molecules in the absence of any influence from the medium polarization. In Refs. [33,37] it has been shown that the dielectric loss spectrum in this case can be conveniently presented as 


$$
\varepsilon_{2}^{\text {micro }}(v)=\varepsilon_{2}(v) \theta(v),
$$

where $\varepsilon_{2}^{\text {micro }}(v)$ and $\varepsilon_{2}(v)$ are the spectra of the imaginary part of the complex microscopic and macroscopic permittivity of the dielectric material particles, respectively. From here, we will consider the latter to be equal to the dielectric permittivity of the bulk medium $\varepsilon_{2}^{b u l k}(v)$. In this case, the Lorentz correction factor, $\theta(v)$, for an isotropic homogeneous medium, expressed as a function of frequency, has the following form

$$
\theta(v)=\left|E(v) / E_{e f f}(v)\right|^{2}=9 /|\varepsilon(v)+2|^{2}
$$

Here $\varepsilon(v)=\varepsilon_{1}(v)-i \varepsilon_{2}(v)$ is the spectral behaviour of the complex dielectric permittivity of the condensed matter in the region of the absorption band considered. The maximum of the function $\theta(v)$, and hence of $\varepsilon_{2}^{\text {micro }}(v)$, is situated near the frequency $v_{F}$ satisfying Frohlich's condition $\operatorname{Re} \varepsilon\left(v_{F}\right)=-2$ [38]. Note that $v_{F}$ is sometimes referred to as Fröhlich's frequency.

The DEP method demonstrates good agreement between the frequency maxima of $\varepsilon_{2}^{\text {micro }}(v)$ spectra and the intrinsic frequencies of the lattice vibrations, calculated from the elastic characteristics of the crystal $[39,40]$. When the local field is taken into account using the Lorentz model, it results in an oscillation frequency of spherically shaped micro-regions of crystal with dimensions far larger than the lattice constant, but much smaller than the wavelength of the probe radiation. As shown in [33], this conclusion applies to all dielectric media, including liquids. Thus, it can be concluded that expression (6) corresponds to the spectrum of individual spherical particles, having their own spectral characteristics different from the characteristics of bulk samples of the same material. These particles can be considered "meso-oscillators" or meso-particles (see Refs. [33,37]), the optical properties of which, in the longwavelength limit $(d<<\lambda)$, originate in spectral differences between the macroscopic and microscopic electromagnetic fields. In this case, the applicability of the concepts underlying the DEP method has been confirmed by results of an analysis of the influence of dipole-dipole interactions on the absorption spectra of granular films of noble metals and their colloidal solutions [17].

\section{DISCUSSION AND RESULTS}

As shown in Ref.[17], allowing for the dispersion of the effective field in the quasi-static approximation enables linkage of the spectral characteristics of small metal particles with the optical properties of bulk metals. The dielectric loss spectrum of the strongly diluted composite medium, consisting of spherical metal particles with a diameter $d<<\lambda$ and a transparent matrix with a permittivity of $\varepsilon_{h}$, can be represented by an expression analogous to Eqn. (6)

$$
\varepsilon_{2}^{\text {micro }}(v)=q \cdot \varepsilon_{2}^{\text {bulk }}(v) \theta(v),
$$

where $\varepsilon_{2}^{\text {bulk }}(v)$ is the dielectric loss spectrum of the bulk metal and $q$ its bulk concentration. The local field factor $\theta(v)$ is given by

$$
\theta(v)=9 /\left|\varepsilon_{2}^{b u l k}(v)+2 \varepsilon_{h}\right|^{2}
$$

where $\varepsilon_{h}$ is the dielectric constant of the surrounding medium. Note that expression (7), with $q<<1$ is consistent with Maxwell-Garnett's formula underlying modern effective media theory. For $q=1$ and $\varepsilon_{h}=$ 1 it coincides with Eqn. (6). The spectrum $\varepsilon_{2}^{\text {micro }}(v)$ in the general case can be considered as a spectrum characteristic of plasmons localized on an isolated spherical metal particle in a medium with permittivity $\varepsilon_{h}$. From an understanding of IMI spectroscopy [32] used in Ref. [17], the oscillator strength of the $j$-th quantum transition in a condensed medium can be represented as

$$
f_{c}=\frac{2 m}{N e^{2}} \cdot v_{c} \int \varepsilon_{2}^{\text {micro }}(v) d v=\frac{2 m}{N e^{2}} \cdot v_{\max } \theta_{a v} \int \varepsilon_{2}^{b u l k}(v) d v=F_{\text {macro }} \theta_{a v}
$$

Here $f_{c}$ is the strength of microscopic oscillators for this transition, $N$ is their concentration, $\theta_{a v}$ is the average value of the effective field factor, equal to the ratio of the integrated intensities of the spectra $\varepsilon_{2}^{\text {micro }}(v)$ and $\varepsilon_{2}^{b u l k}(v)$ and $v_{c}$ and $v_{\max }$ are their respective frequency maxima. The average value of $\theta_{a v}$ can also be determined if, in Eqns. (6) and (7), the spectral value of $\varepsilon_{2}^{b u l k}(v)$ is replaced by the average background value of the dielectric constant in this spectral region, $\varepsilon_{b g}$, and $\varepsilon_{h}$ is set equal to 1 .

For a strong, isolated absorption band, it is a good approximation, however, to use the value of the dielectric constant in the high frequency region of the spectrum, $\varepsilon_{\infty}$, as its average value, following 
from Eqn. (4a). The strength of the microscopic oscillator in Eqn. (9), which is directly dependent on the square of the matrix element of the transition dipole moment $\left(f=\left(m / e^{2}\right) \cdot|\mathrm{d} \mu / \mathrm{d} q|^{2}\right)$, can be determined from the integrated intensity of the spectrum of the imaginary part of the microscopic dielectric permittivity $\varepsilon_{2}^{\text {micro }}(v)$, which is related to $\varepsilon_{2}^{\text {bulk }}(v)$ by Eqn. (6). It can be assumed that, for metals, these microscopic oscillators are electrons, forming a plasmon localized in a spherical nanoparticle. Thus, if we know the value of the average dielectric constant of the metal at frequencies $v$ much larger than the frequency of the localized plasmon, then the strength of the microscopic oscillator is not difficult to assess using Eqn. (4). These evaluations were made using data from dispersion analysis for $\mathrm{Au}, \mathrm{Ag}$ and $\mathrm{Cu}$, reported in Ref. [24].

From the data reported in [17] it follows that similar results can be obtained by analysing the influence of the dielectric permittivity of the surrounding medium, $\varepsilon_{h}$, on the frequency of the isolated plasmons in accordance with Eqn. (7). It has been shown that the shift $\left(\Delta v_{\text {ind-res }}\right)$ of the frequency maximum, $v_{\max }$, of the plasmon absorption band of isolated nanoparticles due to the influence of $\varepsilon_{h}$ can be estimated using

$$
\Delta v_{\text {ind-res }}=v_{\max }\left(\varepsilon_{2}^{\text {micro }}(v) ; \varepsilon_{h}=1\right)-v_{\max }\left(\varepsilon_{2}^{\text {micro }}(v) ; \varepsilon_{h}>1\right)
$$

where the first term on the right-hand side of Eqn. (10) is the peak position of spectrum $\varepsilon_{2}^{\text {micro }}(v)$ at $\varepsilon_{h}$ $=1$, while the second term in this equation is the peak position of that spectrum at $\varepsilon_{h}>1$. According to [17], for the analytical determination of the shift of the plasmon absorption spectra of highly dilute composites of small metal particles caused by inductive-resonant interactions, one can use the following expression

$$
\Delta v_{\text {ind-res }}=\frac{\left(\varepsilon_{h}-1\right)}{6} \cdot\left[\frac{f e^{2}}{\left(4 \pi m c^{2} v_{o} R^{3}\right)}\right]=D \cdot A,
$$

where the factor in the square brackets is determined by the electronic oscillator strength, $f$, in this plasmon, $m$ is the electronic mass, $c$ is the speed of light in vacuum, $v_{o}$ is the frequency maximum of the absorption band and $R$ is the effective radius of the IMI, which correlates with the size of the volume attributable to one electronic oscillator. The concentration of these oscillators is assumed to be equal to the concentration of free electrons in the bulk metal.

From Eqn. (11), it follows that the frequency shift, $\Delta v$, is proportional to the strength of the electronic oscillator and increases linearly with the $\varepsilon_{h}$ of the environment surrounding the particle, a result of the increase in the dielectric factor $D=\left(\varepsilon_{h}-1\right) / 6$. This is supported by results from calculations of the absorption spectra of an isolated plasmon for $\mathrm{Au}$ and $\mathrm{Ag}$, performed in [17], based on Eqns. (7), (10) and (11) as well as by experimental data from Refs. [41,42]. From Eqn. (11), the quantity $A=\Delta v_{\text {ind-res }} / D$, defining in this case the strength of the electronic oscillator of the plasmon, does not depend on the nature of the nanoparticle's environment. Indeed, the calculated value of $A$ in the case of gold and silver, with an increase in the $\varepsilon_{h}$ values from 1.7 to 9 , deviates from the mean value by not more than $10 \%$. Similar linear relationships $\left(\Delta v_{\text {ind-res }}=A \cdot D\right)$ over a narrower range of $\varepsilon_{h}$ values were also obtained for many other metals, and will be published elsewhere. The average values of $A$ for gold and silver, calculated in [17] in this manner from data on the optical constants given in Ref. [43] are $A^{\mathrm{Au}}=4610 \mathrm{~cm}^{-1}$ and $A^{\mathrm{Ag}}=15100$ $\mathrm{cm}^{-1}$ respectively. We note that results for the value of $A$ for gold and silver were recently updated using experimental data on the optical constants of $\mathrm{Au}$ [44] and $\mathrm{Ag}$ [45]. The values obtained are $A^{\mathrm{Au}}=4900$ $\mathrm{cm}^{-1}$ and $A^{\mathrm{Ag}}=15000 \mathrm{~cm}^{-1}$ (see Table 1). These updated results for Au and Ag deviate by less than 5\% from our previously calculated values of $A$, based on $n(v)$ and $k(v)$ from Ref. [43]. Calculations for $\mathrm{Cu}$ were also performed based on tabulated data from Ref. [43], resulting in a value of $A^{\mathrm{Cu}}=3040 \mathrm{~cm}^{-1}$ with calculated spectra $\varepsilon_{2}^{\text {micro }}(v)$ shown for different $\varepsilon_{h}$ values in Fig. 1a and the dependence of $A$ vs $D$ demonstrated in Fig. 1b. We note that more recent results on the optical constants for $\mathrm{Cu}$, published in Ref. [46] (specifically Figure 2 in the paper) seem to be in relatively good agreement with results from [43] over the spectral range investigated.

It follows from Eqn. (11) that an expression for the strength of the electronic oscillator in an isolated plasmon can be expressed as

$$
f=A m 4 \pi^{2} c^{2} v_{o} R^{3} / e^{2} .
$$

Since the concentration of free electrons in nanoparticles of $\mathrm{Au}, \mathrm{Ag}$ and $\mathrm{Cu}$ is equal to the concentration of atoms in the bulk metal and assuming that the radius of the volume, $V$, per electron and the effective radius $R$ of IMI are similar, expression (12) can be transformed to

$$
f=3 A m \pi c^{2} v_{o} / N e^{2}
$$


where $N=1 / N=3 / 4 \pi R^{3}$.

The results of a comparison of electronic oscillator strength for localized plasmons in nanoparticles of gold, silver and copper obtained from Eqn. (13) and corresponding values from Eqn. (4) are shown in Table I. Also shown are results from Ref. [24] on the strength of dispersion oscillators, $F_{0}$, describing the oscillations of free electrons in the corresponding bulk metals and average values of the effective field factors, $\theta_{a v}$, calculated using Eqn. (4a).

From Table I, the oscillator strength of electrons in a spherical nanoparticle or in a localized plasmon for gold, silver and copper, determined from Eqn. (13) and following, according to Eqn. (4), from the dispersion analysis data of Ref. [24], are well correlated (cf. columns VI and VIII). We conclude that the difference in the oscillator strengths of free electrons in the bulk metal and electrons in a spherical nanoparticle in the long-wavelength limit is due to background polarization, occurring at the expense of high-frequency excitation of bound electrons.

Let us now consider the spectral differences in the macro- and micro-characteristics of the metals.

From an analysis of Eqns. (3b) and (5), it has been shown that the basis of the difference in the frequency maximum of the absorption bands for macro- and micro- characteristics in the case considered may be resonant dipole-dipole interactions in the metal environment. As shown in [17], the difference between the dispersion and intrinsic frequencies characterising the macro- and micro-characteristics of condensed matter is determined by the resonant frequency shift, $\Delta v_{d y n}$, of the microscopic oscillator with respect to the frequency maximum of the absorption band of the bulk media:

$$
\Delta v_{d y n}=v_{\max }\left(\varepsilon_{2}^{\text {micro }}(v)\right)-v_{\max }\left(\varepsilon_{2}^{\text {bulk }}(v)\right) .
$$

It is obvious that the resonant shift with respect to the metal, as described by the oscillator model and the dipole approximation, and according to Eqns. (5) and (14) equal to the intrinsic frequency of the plasmon $v_{c}$, can be represented as

$$
\Delta v_{d y n}=v_{c}=\sqrt{\frac{N f_{c} e^{2} \cdot\left(\varepsilon_{\infty}+2\right)}{3 \pi m c^{2} \cdot 3}},
$$

where $f_{c}=F_{0} \theta(v)$ is the strength of the microscopic oscillator, characterising the absorption of free electrons, $v_{\mathrm{c}}$ is the intrinsic frequency, corresponding to the maximum of the $\varepsilon_{2}^{\text {micro }}(v)$ spectrum and $N, e$ and $m$ are the concentration, charge and effective mass of a free electron.

The spectra of experimental optical constants $n(v)$ and $k(v)$ of $\mathrm{Au}, \mathrm{Ag}$ and $\mathrm{Cu}$ according to [4345], as well as the calculated spectra of the plasmon absorption $\varepsilon_{2}^{\text {micro }}(v)$ from Eqn.(6), the local field factor, $\theta(v)$ from Eqn. (6a), and $\varepsilon_{2}^{\text {bulk }}(v)=2 n(v) k(v)$, are presented in Figs. 2a,b,c. The spectra of optical constants, calculated from the Eqn. (3a) by means of parameters $v_{0}, F_{0}$ and $\gamma_{0}$ from Ref. [24] for free electrons, are denoted as $n^{0}(v)$ and $k^{0}(v)$. Using these spectra, the calculated values of the macroscopic and microscopic dielectric permittivities are $\varepsilon_{1}^{0}(v), \varepsilon_{2}^{0}(v)$ and $\varepsilon_{2}^{0 m i c}(v)$ respectively. These spectra are shown in Figs. 2d,e,f.

The peak positions of spectra $\varepsilon_{2}^{\text {micro }}(v)$ (denoted as $v_{c}$ ), $\varepsilon_{2}^{0 m i c}(v)$ (denotes as $v_{c}^{0}$ ) as well as the frequency shift of the localised plasmon from the frequency of the free electrons in the bulk metal, calculated using the theory of resonant interactions by Eqn. (15), are shown in Table 2. Also given are the parameters of dispersion oscillator of absorption band for free electrons from Ref. [24] (column II) and experimental peak position ( $v_{\text {exp }}$ ) of absorption band of spherical nanoparticles (column III) from different sources [2,49-51].

These data show that the calculated frequency shifts are close to the frequency of localized plasmons, obtained using Eqns. (5) and (6), as well as being close to experimental data on the absorption spectra of colloidal solutions and granular films of noble metals [17,36,41,42]. Note also that the values of the frequencies of localized plasmons, compared with the frequency maximum of the spectrum $\varepsilon_{2}^{\text {micro }}(v)$, coincide with a spread of not more than 5\% with other calculated and experimental data [2] obtained for particles with diameters of 1-20 nm (listed in column III of Table 2), for which the contribution of dynamic polarization to the frequency shift is close to zero.

The primary role of free electrons in the plasmon absorption of metal nanoparticles can be identified by comparing the experimental spectral characteristics of bulk silver, gold and copper obtained in [43-45] and calculated by Eqn. (3a) using dispersion oscillator parameters, modelling the contribution of free electrons to the corresponding dielectric loss spectra. 
As can be seen from figures $2 a, b, c$, the optical characteristics of solid metals in the region of plasmon absorption are strongly influenced by absorption due to low frequency excitation of bound electrons. Nevertheless, the plasmon absorption band of gold and silver nanoparticles, calculated according to Eqn. (5), appears at a frequency close to that observed experimentally (see column III in Table 2). Furthermore, it is close to satisfying Fröhlich's condition $\operatorname{Re} \varepsilon\left(v_{F}\right)=-2$, in accordance with the frequency maximum of function $\theta(v)$, see Table 2. If we neglect the low-frequency electron interband transitions, with the contribution of high frequency transitions taken into account via $\varepsilon_{\infty}$, in accordance with Eqns. (3a), it is clear that the frequency maximum of the plasmon absorption band, caused solely by free electrons, is only insignificantly shifted toward the low-frequency part of the spectrum (Figs. 2d,e,f). Also, comparison of the data presented in Figs. 2a,b,c and 2d,e,f shows that low frequency interband transitions lead to a drastic reduction in both the amplitude and linewidth of both the $\theta(v)$ function and the plasmon band $\varepsilon_{2}^{\text {micro }}(v)$.

It is interesting to note that Fröhlich's condition does not apply to $\mathrm{Cu}$, as evidenced in Table 2 and the experimental spectra $\varepsilon_{1}^{\text {bulk }}(v)$ for $\mathrm{Cu}$, see, for example, Fig. 2 in Ref. [46]. A quantitative explanation for the deviation from the $\varepsilon_{1}^{b u l k}\left(v_{F}\right)=-2$ condition was given already (see, for example, Ref. [2] page 36]). It is ascribed to a large and strongly frequency-dependent contribution from $\varepsilon_{2}^{b u l k}(v)$, due at least partially to the location of interband transitions close by. The approach used here clearly demonstrates that if the bulk dielectric constants $\left(\varepsilon_{1}^{0}(v)\right.$ and $\left.\varepsilon_{2}^{0}(v)\right)$ are calculated based on optical constants $\left(n^{0}(v)\right.$ and $\left.k^{0}(v)\right)$, obtained from the dispersion parameters for a Drude oscillator at $v_{0}=0$ from [24], then Fröhlich's condition is satisfied for $\mathrm{Cu}$ as well as for $\mathrm{Au}$ and $\mathrm{Ag}$ (see column VI in Table 2 and Figs. 2d,e,f) .

\section{CONCLUSIONS}

In conclusion we would like to emphasise that the calculations of noble metal micro-characteristics presented here are made in the quasi-static approximation, which is valid for spherical particles with dimensions much smaller than the wavelength of the electromagnetic radiation. The actual particle size is not taken into account and calculated values are normalized to the number of free electrons per unit volume, which in a nanoparticle is assumed to be equal to the concentration of atoms per unit volume of the metal. The results obtained lead to a conclusion that, using a point-dipole approximation, electron-ion interactions in the noble metals can be considered as the interaction of virtual dipoles, which, against the background of the spectral manifestations of all other energy states, determine the optical characteristics of metals in the visible and IR regions of the spectrum. Support for this conclusion comes from modelling the absorption of free electrons by a dispersion oscillator using the Drude-Lorentz model [5,24]. From this data, the intrinsic frequency of the virtual dipole oscillators practically coincides with the frequencies of the maxima of the plasmon absorption bands of the corresponding spherical particles with a diameter less than $20 \mathrm{~nm}$. The shift of these intrinsic frequencies from the frequency of the macroscopic dispersion oscillator for free electrons $\left(v_{0}=0\right)$ agrees well with estimates made using the theory of resonant dipoledipole interactions. Relevant characteristics of plasmon absorption bands appear under confinement conditions, where particle sizes are much smaller than the wavelength of electromagnetic radiation. Under these conditions, effects of optical polarization on the frequency of the probe radiation, and, consequently, the resonant dipole-dipole interactions, are virtually absent.

Clearly, the difference in the frequency of the resonant absorption of electron oscillators localized in the nanoparticle and the intrinsic frequency of the free electrons in the bulk metal is due to resonant dipole-dipole interactions. Differences in the corresponding oscillator strengths are determined by the background polarization, arising from the excitation of bound electrons in the high frequency region of the spectrum.

We would like to mention that despite the fact that the results and interpretations given here relate only to the particular case of small spherical particles of $\mathrm{Au}, \mathrm{Ag}$ and $\mathrm{Cu}$, they indicate the generality of the spectroscopic manifestations of resonant dipole-dipole interactions, not only in liquids and dielectric crystals, but also in metallic media. Our purpose was simply to draw attention to the fact that interesting results can be obtained based solely on considerations from classical physics and by extending molecular spectroscopy approaches to non-molecular structures. Nevertheless, the rather surprising results obtained with simple approaches require further in-depth theoretical study, to which we want to draw the attention of researchers working in the area of theoretical physics. 


\section{REFERENCES}

[1] C.F. Bohren, D.R. Huffman, Absorption and Scattering of Light by Small Particles, New York, Wiley, 1983.

[2] U. Kreibig, M. Volmer, Optical Properties of Metal Clusters. New York - Berlin - Heidelberg, Springer-Verlag $1995,533 p$.

[3] S.A. Maier, Plasmonics: Fundamentals and Applications. N.Y.: Springer. 2007.

[4] W. Cai, V. Shalaev, Optical Metamaterials. Fundamentals and Applications, Springer, 2010.

[5] V.A.G. Rivera, O.B. Silva, Y. Ledemi, Y. Messaddeq, and E. Marega Jr., Collective Plasmon-Modes in Gain Media. Quantum Emitters and Plasmonic Nanostructures, Springer, 2015, 123 p.

[6] M.B. Ross, C.A. Mirkin, G.C. Schatz, Optical Properties of One-, Two-, and Three-Dimensional Arrays of Plasmonic Nanostructures, J. Phys. Chem. C, 120 (2016) 816-830.

[7] H. Zhang, H.V. Demir, A.O. Govorov, Plasmonic Metamaterials and Nanocomposites with the Narrow Transparency Window Effect in Broad Extinction Spectra, ACS Photon., 1 (2014) 822-832.

[8] J. Perez-Juste, I. Pastoriza-Santos, L.M. Liz-Marzan, P. Mulvaney, Gold nanorods: synthesis, characterisation and applications, Coor. Chem. Rev. , 249 (2005) 1870-1901.

[9] P.K. Jain, X. Huang, I.H. El-Saed, M.A. El-Saed, Noble Metals on the Nanoscale: Optical and Photothermal Properties and some Applications in Imaging, Sensing, Biology and Medicine, Acc. Chem. Res. 41 (2008)15781586.

[10] T.A. Klar, Biosensing with plasmonic nanoparticles, in book "Nanophotonics with Surface Plasmonics" Edts. V.M. Shalaev and S. Kawata, Elsevier, Ch. 8, 2007 (pp. 219-264).

[11] A.G. Brolo, Plasmonics for future biosensors, Nat. Photon., 6 (2012) 709-713.

[12] A. Crut, P. Maioli, N. Del Fatti and F. Vallée, Optical absorption and scattering spectroscopies of single nanoobjects, Chem. Soc. Rev., 43 (2014) 3921-3956; J. Olson, S. Dominguez-Medina, A. Hoggard, L.-Y. Wang, W.-S. Chang, S. Link, Optical characterization of single plasmonic nanoparticles, Chem. Soc. Rev., 44 (2015) 40-57.

[13] S. Schlücker, Surface-Enhanced Raman Spectroscopy: Concepts and Chemical Applications, Angew. Chem. Int. Ed., 53 (2014) 4756-4795.

[14] C. Muehlethaler, M. Leona, J.R. Lombardi, Review of Surface Enganced Raman Scattering Applications in Forensic Science, Anal. Chem., 88 (2016) 152-169.

[15] A. Moores, F. Goenttmann, The Plazmon Band in Nobel Metal Nanoparticles: an Introduction to Theory and Application, New J. Chem., 30 (2006) 1121-1132.

[16] V. Myroshnychenko, J. Rodriguez-Fernandez, I. Pastoriza-Santos, A.M. Funston, C. Novo, P. Mulvaney, L.M. LizMarzan, F.J. Garcia de Abajo, Modeling the optical response of gold nanoparticles, Chem. Soc. Rev. 37 (2008) 1792-1805.

[17] I.I. Shaganov, T.S. Perova, M.V. Mukhina, I.V. Martynenko, A.V. Baranov, A.V. Fedorov, V. Gerard, and Y.K. Gun'ko, Influence of intermolecular interactions on spectroscopic characteristics of metal nanoparticles and thei composites, Phys. Chem. Chem. Phys. 16 (2014) 24536-24548.

[18] C.G. Granqvist and O. Hunderi, Conductivity of inhomogeneous materials: Effective-medium theory with dipoledipole interaction, Phys. Rev. B, 18 (1978) 1554-1561.

[19] F. Wooten, Absorption and Dispersion. Optical Properties of Solids. New York, Academic Press, 1972.

[20] X. Fan, W. Zheng, D.J. Singh, Light scattering and surface plasmons on small spherical particles, Light: Sci. \& Applicat., 3 (2014) e179/1-14.

[21] C.G. Granqvist and O. Hunderi, Optical properties of ultrafine gold particles, Phys. Rev. B, 16 (1977) 3513-3534 C.G. Granqvist, N. Calander, and O. Hunderi, Optical properties of ultrafine silver particles, Solid State Commun., 31 (1979) 249-252; S. Norrman, T. Andersson, C.G. Granqvist and O. Hunderi, Optical absorption in discontinuous gold films, Solid State Commun., 23 (1977) 261-265.

[22] S. Yamaguchi, Theory of the optical properties of very thin inhomogeneous films, J. Phys. Soc. Jpn., 17 (1962) 184-193.

[23] S. Yoshida, T. Yamaguchi, and A. Kinbara, Optical Properties of Aggregated Silver Films, J. Opt. Soc. Am., 61 (1971) 62-69.

[24] A.D. Rakic, A.B. Dijrisik, J.M. Elazar, and M.L. Majewski, Optical properties of metal films for vertical-cavity optoelectronic devices, Appl. Opt., 37 (1998) 5271-5273.

[25] R. Brendel, and D. Bormann, An infrared dielectric function model for amorphous solids, J. Appl. Phys., 71 (1992) $1-6$.

[26] H. Gai, J. Wang, Q. Tian, Modified Debye model parameters of metals applicable for broadband calculations, Appl. Optics, 46 (2007) 2229-2233.

[27] W.S.M. Werner, K. Glantschnig, C.J Ambrosch-Draxl, Phys. Chem. Ref. Data, 38 (2009) 1013-1092.

[28] C. Gong, and M.S. Leite, Noble Metal Alloys for Plasmonics, ACS Photonics, 3 (2016) 507-513.

[29] X. Li, J. Zhu, and B. Wei, Hybrid nanostructures of metal/two-dimensional nanomaterials for plasmon-enhanced applications, Chem. Soc. Rev., 45 (2016) 3145-3187.

[30] W.J. Condell, H.I. Mandelberg, Optical Properties of an Isolated Resonance with Natural or Collision Broadening, J. Opt. Soc. Amer., 54 (1964) 973-980.

[31] I.I. Shaganov and V. S. Libov, Manifestation of dipole-dipole interactions in the absorption spectra of various condensed media, Fiz. Tverd. Tela, 17 (1975) 1749 [Sov. Phys. Solid State, 17 (1975) 1136].

[32] N.G. Bakhshiev, Photophysics of Dipole-Dipole Interactions, St. Petersburg, Izd. SPbGU, 2005, 500 p. 
[33] I.I. Shaganov, T.S. Perova, V.A. Melnikov, S. Dyakov, and K. Berwick, Size effect on the infrared spectra of condensed media under condition of 1D, 2D and 3D dielectric confinement, J. Phys. Chem. C, 114 (2010) 1607116081.

[34] N.G. Bakhshiev, O.P. Girin, and V.S. Libov, Relation between the observed and true absorption spectra of molecules in a solid medium. II. Methods for determining the correction of a universal effect of the effective (internal) field, Opt. Spektrosk., 14 (1963) 745 [Opt. Spectrosc. (USSR), 14 (1963) 634].

[35] V.S. Libov, Advancements and perspectives in use of local field method to study spectral manifestation of resonance interactions in condensed medium, Rus. J. Phys. Chem., 54 (1980) 817.

[36] I.I. Shaganov, Investigation of the effect of the resonant interactions on spectroscopic characteristics of the bands of anomalous plasma absorption of thin metal films, Rus. Opt.\& Spectr., 49 (1980) 181-184.

[37] I.I. Shaganov, T.S. Perova, R.A. Moore, and K. Berwick, Effect of the internal field on the IR absorption spectra of small particles in the case of 3D, 2D and 1D size confinement, J. Phys. Chem. B., 109 (2005) 9885-9891.

[38] H. Fröhlich, Theory of Dielectrics, Oxford University Press London, 1958.

[39] T.S. Tolstykh, I.I. Shaganov, V.S. Libov, Sov. Phys. Solid State, 16 (1974) 657-662.

[40] T.S. Perova, I.I. Shaganov, S. Unnikrishnan, and R.A. Moore, Spectroscopic Characteristics of Nano-Composite Structures in 3D, 2D and 1D Size Confinement, Proceedings SPIE, 5826 (2005) 387-396.

[41] T. Okamoto, I. Yamaguchi, T. Kobayahi, Local plasmon sensor with gold colloid monolayers deposited upon glass substrates, Opt. Lett., 2 (2000) 372-374.

[42] J.J. Mosk, D.R. Smith, S. Shultz, Local refractive index dependence of plasmon resonance spectra from individual nanoparticles, Nano Lett., 3 (2003) 485-491.

[43] P.B. Jonson, and R.W. Christy, Optical constant of the Noble Metals, Phys. Rev. B, 6 (1972) 4370-4379.

[44] R. L. Olmon, B. Slovick, T. W. Johnson, D. Shelton, S.-H. Oh, G. D. Boreman, and M.B. Raschke, Phys. Rev. B, 86 (2012) 235147/1-9.

[45] H. U. Yang, J. D’Archangel, M. L. Sundheimer, E. Tucker, G. D. Boreman, and M. B. Raschke, Phys. Rev. B, 91 (2015) 235137/1-11.

[46] K. Stahrenberg, Th. Herrmann, K. Wilmers, N. Esser, W. Richter and M.J.G. Lee, Phys. Rev. B, 64, (20011) 15111/1-9.

[47] C. Sönnichsen, Plasmons in metal nanostructures, Ph.D. thesis, Munchen, 2001.

[48] P. Winsemius, Temperature dependence of the optical properties of $\mathrm{Au}$ and Ag, Ph.D. thesis, University of Leiden, 1972.

[49] H. Abe, K.P. Charle, B. Tesche and N. Schulze, Surface plasmon absorption of various colloidal metal particles, Chem. Phys., 68 (1982) 137-141.

[50] I. Schick, S. Lorenz, D. Gehrig, S. Tenzer, W. Storck, K. Fischer, D. Strand, F. Laquai and W. Tremel, Inorganic Janus particles for biomedical applications, Beilstein J. Nanotech., 5 (2014) 2346-2362.

[51] K.-J. Berg, A. Berger, and H. Hofmeister, Small silver particles in glass surface layers produced by sodium-silver ion-exchange - their concentration and size depth profile, J. Physik D, 20 (1991) 309-311.

\section{Figures}

Figure 1. Dielectric-loss spectra $\varepsilon_{2}^{\text {micro }}(v)$ of plasmon absorption in spherical particles of $\mathrm{Cu}$ in various matrices, calculated in the quasi-static limit from Eqns. (7),(8) with variation of $\varepsilon_{h}$ of the ambient medium: 1 (a), $2.4(b), 3.5$ $(c), 5(d), 7(e), 9(f)$. b) Linear dependence of the inductive-resonant dipole-dipole frequency shift of the absorption maximum by a plasmon in $\mathrm{Cu}$ vs dielectric factor $D=\left(\varepsilon_{h}-1\right) / 6$. The obtained slope, $A=\Delta v / D$, is equal to $3040 \mathrm{~cm}^{-1}$. The numbers in brackets in Figure a) correspond to the numbers shown beside the relevant curves; the data for curve (1) is multiplied by a factor of 7 and is also shown with curves 2 and 3 in the insert for clarity.

Figure 2. Experimental and calculated spectra of optical characteristics of noble metals. Calculations of spectra $\varepsilon_{2}^{\text {bulk }}(v)=2 n(v) k(v), \theta(v)$ (using Eqn. (6a)), and $\varepsilon_{2}^{\text {micro }}(v)$ (using Eqn. (6)) are based on experimental spectra $n(v)$ and $k(v)$ taken from a) Ref. [44] for Au, b) Ref. [45] for Ag and c) Ref. [43] for Cu. The real $\varepsilon_{1}^{0}(v)$ and imaginary $\varepsilon_{2}^{0}(v)$ parts of dielectric function $\varepsilon^{b u l k}(v)$, calculated by Eqn.(3a) for the bulk metal based on parameters $v_{0}, F_{0}$ and $\gamma_{0}$ from Ref. [24] for zero oscillator, and calculated by Eqn. (6) using these functions, spectra $\varepsilon_{2}^{0 m i c}(v)$ for $\mathrm{Au}(\mathrm{d}), \mathrm{Ag}(\mathrm{e})$ and $\mathrm{Cu}(\mathrm{f})$. The peak position of spectrum $\varepsilon_{2}^{0 m i c}(v)$, and the intersection with spectrum of $\varepsilon_{1}^{0}(v)$, correspondent to Fröhlich's condition $\operatorname{Re} \varepsilon\left(v_{F}\right)=-2$, are shown by number beside each spectrum and by arrow in figures d), e), and f). For clarity of presentations, some of the spectra are multiplied by a specific factor shown in the legends. 
Figure 1
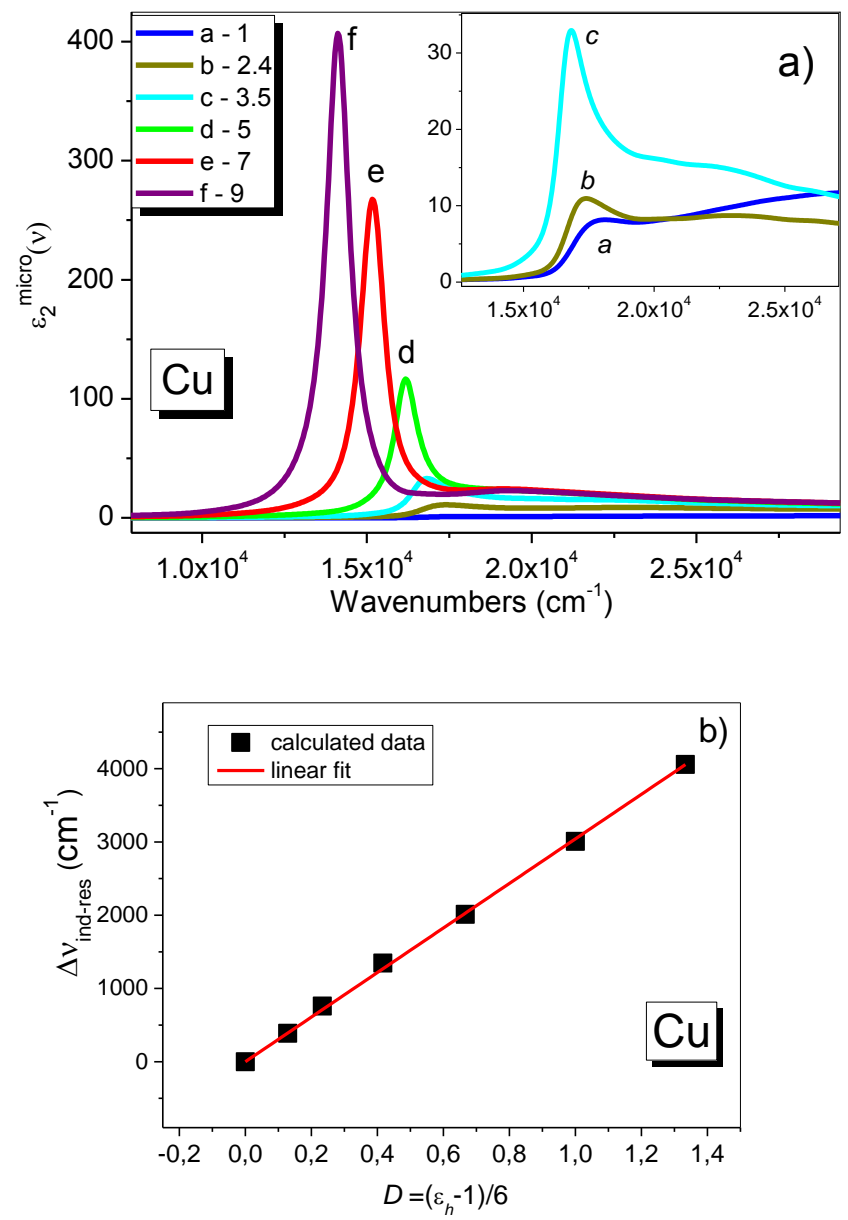
Figure 2.

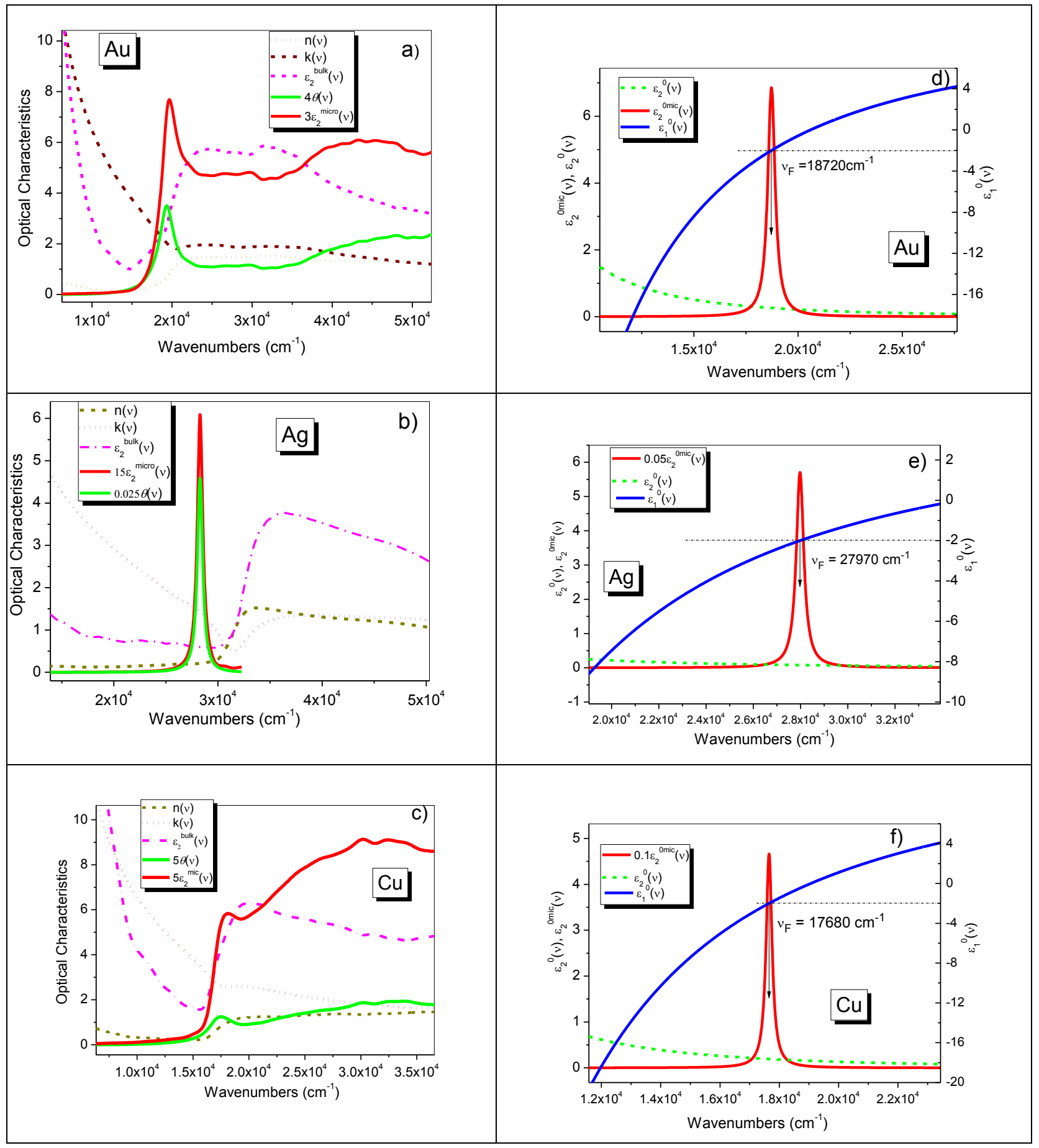


Table 1. Comparison of the electronic oscillator strength for localized plasmons in nanoparticles of Au, $\mathrm{Ag}$ and $\mathrm{Cu}$, reported in Ref. [24] and obtained from Eqn. (14) for free electron oscillators, and calculated from experimental optical constants of bulk metal using Eqn. (13).

\begin{tabular}{|c|c|c|c|c|c|c|c|c|}
\hline Metal & $\begin{array}{l}N \mathrm{x} 10^{22}, \\
\mathrm{~cm}^{-3}\end{array}$ & $\varepsilon_{\infty}$ & $\begin{array}{l}\theta_{a v} \\
\text { Eqn.(4a) }\end{array}$ & $F_{0}$ & $\begin{array}{l}f=F_{0} \theta_{a v} \\
\text { Eqn. (4) }\end{array}$ & $A, \mathrm{~cm}^{-1}$ & $\begin{array}{c}f \\
\text { Eqn. (13) }\end{array}$ & $\begin{array}{c}v_{c} \mathrm{~cm}^{-1} \\
\text { peak position } \\
\text { of } \varepsilon_{2}^{\text {micro }}(v) \\
\text { at } \varepsilon_{h}=1\end{array}$ \\
\hline I & II & III & IV & V & VI & VII & VIII & IX \\
\hline $\mathrm{Au}$ & 5.9 & $9.5^{\mathrm{a}}$ & 0.068 & $\begin{array}{l}0.76^{\mathrm{b}} \\
0.77^{\mathrm{c}}\end{array}$ & $\begin{array}{l}0.0517 \\
0.0524\end{array}$ & $\begin{array}{l}4610^{\mathrm{d}} \\
4900^{\mathrm{e}}\end{array}$ & $\begin{array}{l}0.0512^{\mathrm{d}} \\
0.0551^{\mathrm{e}}\end{array}$ & $\begin{array}{l}19610 \\
19840\end{array}$ \\
\hline $\mathrm{Ag}$ & 5.86 & $3.7[48]$ & 0.277 & $\begin{array}{l}0.845^{\mathrm{b}} \\
0.821^{\mathrm{c}}\end{array}$ & $\begin{array}{l}0.234 \\
0.227\end{array}$ & $\begin{array}{l}15100^{\mathrm{d}} \\
15000^{\mathrm{f}}\end{array}$ & $\begin{array}{l}0.241^{\mathrm{d}} \\
0.242^{\mathrm{f}}\end{array}$ & $\begin{array}{l}28170 \\
28250\end{array}$ \\
\hline $\mathrm{Cu}$ & 8.49 & $\begin{array}{c}12.07 \\
{[47]}\end{array}$ & 0.0454 & $\begin{array}{l}0.575^{b} \\
0.562^{\mathrm{b}}\end{array}$ & $\begin{array}{c}0.026 \\
0.0255\end{array}$ & 3040 & 0.022 & 18180 \\
\hline
\end{tabular}

${ }^{\mathrm{a}} \varepsilon_{\infty}=9.5$ corresponds to the average value calculated using data from Refs. $[26,47,48]$

${ }^{\mathrm{b}}$ Parameters of Drude-Lorentz dispersion oscillator from Ref. [24]

${ }^{c}$ Parameters of Brendel-Bormann dispersion oscillator from Ref. [24]

${ }^{\mathrm{d}}$ Calculated using optical constants from [43]

e Calculated using optical constants from [44]

${ }^{\mathrm{f}}$ Calculated using $n(v)$ and $k(v)$ from [45]

Table 2. Comparison of peak positions of isolated plasmons of $\mathrm{Au}, \mathrm{Ag}$ and $\mathrm{Cu}$, obtained in the quasistatic approximation using experimental optical constants $(n(v)$ and $k(v))$ from Refs. [43-45], as well as from dispersion parameters for the absorption band of free electrons from Ref. [24] and the theory of intermolecular interactions using Eqn. (15).

\begin{tabular}{|c|c|c|c|c|c|c|c|c|c|}
\hline \multirow{2}{*}{ Metal } & \multirow{2}{*}{\multicolumn{3}{|c|}{$\begin{array}{l}\text { Parameters of dispersion } \\
\text { oscillator of absorption } \\
\text { band for free electrons from } \\
\text { Ref. [24] }\end{array}$}} & \multirow{2}{*}{$\begin{array}{l}\text { Exp. peak } \\
\text { position of } \\
\text { abs. band of } \\
\text { spherical } \\
\text { nano- } \\
\text { particles, } \\
v_{\text {exp }}, \mathrm{cm}^{-1}\end{array}$} & \multirow{2}{*}{$\begin{array}{l}v_{\max } \text {, peak } \\
\text { position of } \\
\varepsilon_{2}^{\text {micro }}(v) \\
\mathrm{cm}^{-1} \\
(\text { Eqn. }(6) \\
\text { and data } \\
\text { from [43- } \\
46])\end{array}$} & \multirow{2}{*}{$\begin{array}{l}\text { Peak } \\
\text { position of } \\
\text { abs. spectra } \\
\text { of free } \\
\text { electrons } \\
\text { Eqn.(15) } \\
v_{c}, \mathrm{~cm}^{-1}\end{array}$} & \multicolumn{2}{|c|}{ Fröhlich's frequency } & \multirow{2}{*}{$\begin{array}{l}v_{\mathrm{c}}^{0}, \text { of } \\
\text { spectra } \\
\varepsilon_{2}^{0 m i c}(v) \\
\text { obtained } \\
\text { for 0-os- } \\
\text { cillator, } \\
\mathrm{cm}^{-1}\end{array}$} \\
\hline & & & & & & & $\begin{array}{l}\text { From 0- } \\
\text { oscillator } \\
\text { for } \varepsilon 1^{0}\left(v_{F}\right), \\
v_{F}^{0}, \mathrm{~cm}^{-1}\end{array}$ & $\begin{array}{l}\text { From bulk } \\
\text { for } \\
\varepsilon_{1}^{\text {bulk }}\left(v_{\mathrm{F}}\right) \\
v_{F}^{\text {bulk }}, \mathrm{cm}^{-1}\end{array}$ & \\
\hline \multirow[t]{2}{*}{ I } & \multicolumn{3}{|c|}{ II } & III & IV & $\mathrm{V}$ & VI & VII & VIII \\
\hline & $\omega_{0}{ }^{\mathrm{a}}$ & $F_{0}$ & $\Gamma_{0}^{\mathrm{a}}$ & & & & & & \\
\hline $\mathrm{Au}$ & 0 & 0.76 & 0.053 & $\begin{array}{c}19530^{\mathrm{b}} \\
19417^{\mathrm{c}} \\
19380^{\mathrm{e}} \\
<19442>^{*}\end{array}$ & $\begin{array}{c}19610 \\
{[43]} \\
19840 \\
{[44]}\end{array}$ & 18560 & 18720 & $\begin{array}{c}19763 \\
{[44]}\end{array}$ & 18720 \\
\hline $\mathrm{Ag}$ & 0 & 0.845 & 0.048 & $\begin{array}{c}27027^{\mathrm{b}} \\
24875^{\mathrm{d}} \\
28248^{\mathrm{e}} \\
\langle 26717\rangle^{*}\end{array}$ & $\begin{array}{c}28170 \\
{[43]} \\
28250 \\
{[45]} \\
\end{array}$ & 28380 & 27970 & $\begin{array}{c}28249 \\
{[45]}\end{array}$ & 27980 \\
\hline $\mathrm{Cu}$ & 0 & 0.575 & 0.03 & $\begin{array}{l}17857^{\mathrm{b}} \\
18148^{\mathrm{e}}\end{array}$ & $\begin{array}{c}18180 \\
{[46]}\end{array}$ & 16200 & 17680 & $\begin{array}{c}27420 \\
{[46]}\end{array}$ & 17650 \\
\hline
\end{tabular}




\begin{tabular}{|l|l|l|l|l|l|l|l|l|l|}
\hline & & & & $<18003>^{\mathrm{f}}$ & & & & & \\
\hline
\end{tabular}

${ }^{\text {a }}$ Data from Ref. [24] in eV.

${ }^{b}$ Data from Ref. [49] (see also Fig. 4.13 from Ref.[2]).

${ }^{\mathrm{c}}$ Data from Ref. [50].

${ }^{\mathrm{d}}$ Data from Ref. [51] (see also Ref. [2], Fig. 2.42).

${ }^{\mathrm{e}}$ Calculation data from Ref. [2] (see Appendix A, pages 452-455).

${ }^{\mathrm{f}}$ Average value from the data above. 